\title{
On Weak Tail Domination of Random Vectors
}

\author{
by \\ Rafał LATAEA \\ Presented by Stanistaw KWAPIEN
}

Summary. Motivated by a question of Krzysztof Oleszkiewicz we study a notion of weak tail domination of random vectors. We show that if the dominating random variable is sufficiently regular then weak tail domination implies strong tail domination. In particular, a positive answer to Oleszkiewicz's question would follow from the so-called Bernoulli conjecture. We also prove that any unconditional logarithmically concave distribution is strongly dominated by a product symmetric exponential measure.

1. Introduction. This note is inspired by the following problem about Rademacher series, posed by Krzysztof Oleszkiewicz (private communication):

Problem. Suppose that $\left(\varepsilon_{i}\right)$ is a Rademacher sequence (i.e. a sequence of independent symmetric \pm 1 r.v.'s) and $x_{i}, y_{i}$ are vectors in some Banach space $F$ such that the series $\sum_{i} x_{i} \varepsilon_{i}$ and $\sum_{i} y_{i} \varepsilon_{i}$ are a.s. convergent and

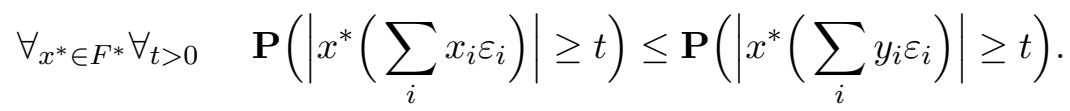

Does this imply that

$$
\mathbf{E}\left\|\sum_{i} x_{i} \varepsilon_{i}\right\| \leq L \mathbf{E}\left\|\sum_{i} y_{i} \varepsilon_{i}\right\|
$$

for some universal constant $L<\infty$ ?

Motivated by the above question we introduce a notion of weak tail domination of random vectors. We prove that if the dominating vector has a regular distribution (including the Gaussian case), then weak tail domination yields strong tail domination (Theorem 1). In particular, Oleszkiewicz's

2000 Mathematics Subject Classification: Primary 60E15; Secondary 60G50, 52A40.

Key words and phrases: sums of independent random variables, Bernoulli series, random vectors, log-concave distributions. 
question has a positive answer provided that the so-called Bernoulli conjecture holds. We also show that in general weak tail domination does not yield comparison of means or medians of norms, even if the distribution of the dominated vector is Gaussian.

In the last part we use Theorem 1 to extend the result of Bobkov and Nazarov [3] and show that unconditional logarithmically concave distributions are strongly dominated by a product symmetric exponential measure.

2. Weak and strong tail domination. Let us begin with the following definition.

Definition 1. Let $X$ and $Y$ be random vectors with values in some Banach space $F$. We say that tails of $Y$ are weakly dominated by tails of $X$ and write $Y \prec_{\omega} X$ if

$$
\mathbf{P}\left(\left|x^{*}(Y)\right| \geq t\right) \leq \mathbf{P}\left(\left|x^{*}(X)\right| \geq t\right) \quad \text { for all } x^{*} \in F^{*}, t>0 .
$$

The following regularity property of random vectors will give us a tool to go from weak to strong comparison. Its roots are in the paper [8], where a modification of the majorizing measure idea was introduced for processes controlled by a family of distances.

Definition 2. We say that a random vector $X$ with values in $F$ is $K$-regular for some $K<\infty$ if there exists a sequence $\left(x_{n}^{*}\right) \subset F^{*}$ such that $\left\|x_{n}^{*}(X)\right\|_{\log (n+2)}=\left(\mathbf{E}\left|x_{n}^{*}(X)\right|^{\log (n+2)}\right)^{1 / \log (n+2)} \leq K \mathbf{E}\|X\|$ for $n=1,2, \ldots$ and

$$
B_{F^{*}}=\left\{x^{*} \in F^{*}:\left\|x^{*}\right\| \leq 1\right\} \subset \operatorname{cl}_{X}\left(\operatorname{conv}\left\{ \pm x_{n}^{*}: n \geq 1\right\}\right),
$$

where for $A \subset F^{*}, \operatorname{cl}_{X}(A)$ denotes the closure of $A$ with respect to the $L^{2}$ distance $d_{X}\left(x^{*}, y^{*}\right):=\left(\mathbf{E}\left|x^{*}(X)-y^{*}(X)\right|^{2}\right)^{1 / 2}$.

Proposition 1. If $X$ is $K$-regular and $Y \prec_{\omega} X$, then

$$
\mathbf{E}\|Y\| \leq 20 K \mathbf{E}\|X\| \text {. }
$$

Proof. Let $x_{n}^{*}$ be as in Definition 2. For any $t>0$ we have

$$
\begin{aligned}
\mathbf{P}\left(\sup _{n \geq 1}\left|x_{n}^{*}(Y)\right| \geq t\right) & \leq \sum_{n \geq 1} \mathbf{P}\left(\left|x_{n}^{*}(Y)\right| \geq t\right) \leq \sum_{n \geq 1} t^{-\log (n+2)} \mathbf{E}\left|x_{n}^{*}(Y)\right|^{\log (n+2)} \\
& \leq \sum_{n \geq 1} t^{-\log (n+2)} \mathbf{E}\left|x_{n}^{*}(X)\right|^{\log (n+2)} \\
& \leq \sum_{n \geq 1}\left(\frac{K \mathbf{E}\|X\|}{t}\right)^{\log (n+2)} .
\end{aligned}
$$


Notice that $d_{Y}\left(x^{*}, y^{*}\right) \leq d_{X}\left(x^{*}, y^{*}\right)$, hence $B_{F}^{*}$ is also contained in the closure of the absolute convex hull of $\pm x_{n}^{*}$ in the $d_{Y}$ metric and thus

$$
\begin{aligned}
\mathbf{E}\|Y\| & \leq \mathbf{E} \sup _{n \geq 1}\left|x_{n}^{*}(Y)\right| \leq K \mathbf{E}\|X\|\left(e^{2}+\int_{e^{2}}^{\infty} \mathbf{P}\left(\sup _{n \geq 1}\left|x_{n}^{*}(Y)\right| \geq t K \mathbf{E}\|X\|\right) d t\right) \\
& \leq K \mathbf{E}\|X\|\left(e^{2}+\sum_{n=1}^{\infty} \int_{e^{2}}^{\infty} t^{-\log (n+2)} d t\right) \leq 20 K \mathbf{E}\|X\| . \text { - }
\end{aligned}
$$

THeOREM 1. Let $X_{1}, X_{2}, \ldots$ be independent copies of a symmetric random vector $X$. Suppose that there exist constants $K<\infty$ and $\alpha, \beta>0$ such that for all $n=1,2, \ldots$,

(i) the random vector $\left(X_{1}, \ldots, X_{n}\right)$ with values in $l_{\infty}^{n}(F)$ is $K$-regular,

(ii) $\mathbf{P}\left(\max _{i \leq n}\left\|X_{i}\right\| \geq \alpha \mathbf{E} \max _{i \leq n}\left\|X_{i}\right\|\right) \geq \beta$.

Then for any random vector $Y$ such that $Y \prec_{\omega} X$ we have, for all $\varepsilon>0$,

$$
\mathbf{P}(\|Y\| \geq t) \leq \frac{2}{\beta} \mathbf{P}\left(\|X\| \geq \frac{\alpha t}{80 K}\right) .
$$

The main idea of how to derive comparison of tails from comparison of means is not new - it goes back at least to the paper of Asmar and Montgomery-Smith [1].

Proof. We may obviously assume that $Y$ is symmetric; we will denote by $Y_{1}, Y_{2}, \ldots$ independent copies of $Y$. Let $n \geq 2$ be such that

$$
2 / n \geq \mathbf{P}(\|Y\| \geq t) \geq 1 / n .
$$

Then $\mathbf{P}\left(\max _{i \leq n}\left\|Y_{i}\right\| \geq t\right) \geq 1-(1-1 / n)^{n} \geq 1 / 2$, hence $\mathbf{E} \max _{i \leq n}\left\|Y_{i}\right\| \geq$ $t / 2$. Let $\eta$ be a r.v. independent of $\left(Y_{i}\right)$ such that $\mathbf{P}(\eta=1)=\mathbf{P}(\eta=0)=$ $1 / 2$. Then by Theorem 3.2.1 of [5], $\eta\left(Y_{1}, \ldots, Y_{n}\right) \prec_{\omega}\left(X_{1}, \ldots, X_{n}\right)$, where both sides are considered as random vectors in $l_{\infty}^{n}(F)$. By Proposition 1,

$$
\begin{aligned}
\frac{t}{4} & \leq \mathbf{E} \max _{i \leq n}\left\|\eta Y_{i}\right\|=\mathbf{E}\left\|\eta\left(Y_{1}, \ldots, Y_{n}\right)\right\|_{l_{\infty}^{n}(F)} \leq 20 K \mathbf{E}\left\|\left(X_{1}, \ldots, X_{n}\right)\right\|_{l_{\infty}^{n}(F)} \\
& =20 K \mathbf{E} \max _{i \leq n}\left\|X_{i}\right\| .
\end{aligned}
$$

Property (ii) yields

$$
\begin{aligned}
& \beta \leq \mathbf{P}\left(\max _{i \leq n}\left\|X_{i}\right\| \geq \frac{\alpha t}{80 K}\right) \leq n \mathbf{P}\left(\|X\| \geq \frac{\alpha t}{80 K}\right), \\
& \text { so } \mathbf{P}(\|X\| \geq \alpha t /(80 K)) \geq \beta / n \geq \beta \mathbf{P}(\|Y\| \geq t) / 2 .
\end{aligned}
$$

REMARK 1. The comparison of the first and second moments of maxima,

$$
\mathbf{E} \max _{i \leq n}\left\|X_{i}\right\|^{2} \leq C\left(\mathbf{E} \max _{i \leq n}\left\|X_{i}\right\|\right)^{2},
$$

implies by the Paley-Zygmund inequality (cf. [5, Lemma 0.2.1]) property (ii) of the previous theorem with $\alpha=1 / 2$ and $\beta=1 /(4 C)$. 
REMARK 2. The proofs of both Proposition 1 and Theorem 1 show that both statements hold if we replace the condition $Y \prec_{\omega} X$ by the condition

$$
\left\|x^{*}(Y)\right\|_{p} \leq\left\|x^{*}(X)\right\|_{p} \quad \text { for all } x^{*} \in F^{*}, p \geq 1 .
$$

Moreover, if $\left\|x^{*}(X)\right\|_{2 p} \leq C\left\|x^{*}(X)\right\|_{p}$ for all $p \geq 1$ and $x^{*} \in F^{*}$ it is enough to check (2) for $p$ being an even integer (the constants $20 K$ and $80 K$ will then change to $20 C K$ and $80 C K$ ).

Let us give a few examples of random vectors satisfying the assumptions of Theorem 1.

EXAmple 1. Any centered Gaussian vector on a separable Banach space is $L$-regular with a universal $L$. This is a consequence of the majorizing measure theorem (cf. [7] and [9, Theorem 2.1.8]). Since a product of Gaussian measures is again Gaussian, property (i) holds with $K=L$. Moments of Gaussian vectors are comparable so by Remark 1 also property (ii) holds with $\alpha=1 / 2$ and a universal $\beta$.

EXAMPLE 2. Let $\left(\eta_{i}\right)$ be a sequence of independent symmetric real r.v.'s with logarithmically concave tails satisfying the $\Delta_{2}$ condition, and let $v_{i} \in F$ be such that $X=\sum_{i} v_{i} \eta_{i}$ is a.s. convergent. Then $X$ is $K$-regular with a constant $K$ depending only on the $\Delta_{2}$ constant ([6, Theorem 3]). The random variable $\left(X_{1}, \ldots, X_{n}\right)$ has an analogous series representation in $l_{\infty}^{n}(F)$, so property (i) holds. It can also be checked that (1) is satisfied with a universal $C$.

REMARK 3. A positive answer to the Bernoulli conjecture ([9, Chapter 4]) would imply the $L$-regularity of Rademacher series. Since (1) holds for $X$ being a Rademacher sum with vector coefficients, Theorem 1 would give a positive answer to Oleszkiewicz's question.

We conclude this section with an example showing that weak tail domination does not yield any comparison of strong parameters even if the dominated vector has Gaussian distribution.

EXAMPLE 3. Let $F=l_{\infty}^{n}, Y=\sum_{i=1}^{n} g_{i} e_{i}$ and $X=9\left(\left|g_{1}\right|+1\right) \sum_{i=1}^{n} \eta_{i} e_{i}$, where $g_{i}$ are i.i.d. $\mathcal{N}(0,1)$ and $\eta_{i}$ are i.i.d. r.v.'s with uniform distribution on $[-1,1]$, independent of $g_{1}$.

To show that tails of $Y$ are weakly dominated by tails of $X$ it is enough to check that

$$
\mathbf{P}(|\langle u, Y\rangle| \geq t) \leq \mathbf{P}(|\langle u, X\rangle| \geq t) \quad \text { for } u \in S^{n-1}, t \geq 0 .
$$

Let us fix $u \in S^{n-1}$. For $t>0$ we have

$$
\mathbf{P}(|\langle u, Y\rangle| \geq t)=\mathbf{P}\left(\left|g_{1}\right| \geq t\right) .
$$


By the Paley-Zygmund inequality,

$$
\begin{aligned}
\mathbf{P}\left(\left|\sum_{i=1}^{n} u_{i} \eta_{i}\right| \geq \frac{1}{3}\right) & =\mathbf{P}\left(\left|\sum_{i=1}^{n} u_{i} \eta_{i}\right|^{2} \geq \frac{1}{3} \mathbf{E}\left|\sum_{i=1}^{n} u_{i} \eta_{i}\right|^{2}\right) \\
& \geq\left(1-\frac{1}{3}\right)^{2} \frac{\left(\mathbf{E}\left|\sum_{i=1}^{n} u_{i} \eta_{i}\right|^{2}\right)^{2}}{\mathbf{E}\left|\sum_{i=1}^{n} u_{i} \eta_{i}\right|^{4}} \geq \frac{4}{27}
\end{aligned}
$$

thus

$$
\mathbf{P}(|\langle u, X\rangle| \geq t) \geq \frac{4}{27} \mathbf{P}\left(3\left(\left|g_{1}\right|+1\right) \geq t\right) \geq \frac{4}{27} \mathbf{P}\left(\left|g_{1}\right| \geq \frac{t}{3}\right) .
$$

Using the easy estimate $2 t \exp \left(-(2 t)^{2} / 2\right) / \sqrt{2 \pi} \leq \mathbf{P}(|g| \geq t) \leq \exp \left(-t^{2} / 2\right)$, we immediately get (3) for $t \geq 3$. For $0 \leq t \leq 3$ we have

$$
\begin{aligned}
\mathbf{P}(|\langle u, X\rangle| \leq t) & \leq \mathbf{P}\left(9\left|\sum_{i=1}^{n} u_{i} \eta_{i}\right| \leq t\right) \leq \frac{\sqrt{2} t}{9} \leq t \frac{\mathbf{P}\left(\left|g_{1}\right| \leq 3\right)}{3} \\
& \leq \mathbf{P}\left(\left|g_{1}\right| \leq t\right)=\mathbf{P}(|\langle u, Y\rangle| \leq t),
\end{aligned}
$$

where to get the second inequality we have used Ball's upper bound on cube sections [2]. Hence (3) also holds for $t \in[0,3]$.

Thus $Y \prec_{\omega} X$. However, $\mathbf{E}\|Y\|=\mathbf{E} \max _{i \leq n}\left|g_{i}\right| \geq \sqrt{\log (n+1)} / L$ and $\mathbf{E}\|X\| \leq 9 \mathbf{E}\left(\left|g_{1}\right|+1\right) \leq 18$.

3. Unconditional logarithmically concave distributions. A Borel probability measure $\mu$ on $\mathbb{R}^{n}$ is called logarithmically concave if for any nonempty compact sets $A, B$ and $\lambda \in[0,1], \mu(\lambda A+(1-\lambda) B) \geq \mu(A)^{\lambda} \mu(B)^{1-\lambda}$. If the support of $\mu$ has full dimension, $\mu$ is log-concave if and only if $\mu$ has the density of the form $e^{-g}$, where $g: \mathbb{R}^{n} \rightarrow(-\infty, \infty$ ] is convex [4]. A random vector $X=\left(X_{1}, \ldots, X_{n}\right)$ is unconditional if for any sequence of signs $s_{i}= \pm 1,\left(s_{1} X_{1}, \ldots, s_{n} X_{n}\right)$ has the same distribution as $X$.

TheOREM 2. Suppose that $Y=\left(Y_{1}, \ldots, Y_{n}\right)$ is an unconditional vector with logarithmically concave distribution such that $\mathbf{E} Y_{i}^{2}=1$, and $X=$ $\left(X_{1}, \ldots, X_{n}\right)$, where the $X_{i}$ are independent, symmetric, exponential random variables with variance 1 (i.e. the density $2^{-1 / 2} \exp (-\sqrt{2}|x|)$ ). Then for any norm on $\mathbb{R}^{n}$,

$$
\mathbf{P}(\|Y\| \geq t) \leq L \mathbf{P}(\|X\| \geq t / L) \quad \text { for } t>0,
$$

where $L$ is a universal constant.

Proof. By Example 2 the vector $X$ satisfies the assumption of Theorem 1 . Moreover, $\left\|\sum_{i} a_{i} X_{i}\right\|_{2 p} \leq C\left\|\sum_{i} a_{i} X_{i}\right\|_{p}$ for $p \geq 1$ and a universal $C$. Thus by Remark 2 it is enough to show that for any $a_{1}, \ldots, a_{n}$ and any positive 
even integer $k$,

$$
\left(\mathbf{E}\left|\sum_{i=1}^{n} a_{i} Y_{i}\right|^{k}\right)^{1 / k} \leq L_{1}\left(\mathbf{E}\left|\sum_{i=1}^{n} a_{i} X_{i}\right|^{k}\right)^{1 / k}
$$

The result of Bobkov and Nazarov [3, Proposition 3.3] gives that for $t_{1}, \ldots, t_{n}$ $\geq 0$,

$$
\mathbf{P}\left(\left|Y_{1}\right| \geq L_{1} t_{1}, \ldots,\left|Y_{n}\right| \geq L_{1} t_{n}\right) \leq \mathbf{P}\left(\left|X_{1}\right| \geq t_{1}, \ldots,\left|X_{n}\right| \geq t_{n}\right),
$$

thus after integration by parts we get

$$
\mathbf{E}\left(\left|Y_{1}\right|^{2 l_{1}} \cdots\left|Y_{n}\right|^{2 l_{n}}\right) \leq L_{1}^{2\left(l_{1}+\cdots+l_{n}\right)} \mathbf{E}\left(\left|X_{1}\right|^{2 l_{1}} \cdots\left|X_{n}\right|^{2 l_{n}}\right),
$$

and (4) immediately follows.

Acknowledgments. The author would like to thank Prof. S. Kwapień for suggesting the method used in the proof of Theorem 1.

This research was partially supported by the Foundation for Polish Science and MEiN Grant 1 PO3A 01229.

\section{References}

[1] N. Asmar and S. Montgomery-Smith, On the distribution of Sidon series, Ark. Mat. 31 (1993), 13-26.

[2] K. Ball, Cube slicing in $R^{n}$, Proc. Amer. Math. Soc. 97 (1986), 465-473.

[3] S. G. Bobkov and F. L. Nazarov, On convex bodies and log-concave probability measures with unconditional basis, in: Lecture Notes in Math. 1807, Springer, Berlin, 2003, 53-69.

[4] C. Borell, Convex set functions in d-space, Period. Math. Hungar. 6 (1975), 111-136.

[5] S. Kwapień and W. Woyczyński, Random Series and Stochastic Integrals: Single and Multiple, Birkhäuser, Boston, 1992.

[6] R. Latała, Sudakov minoration principle and supremum of some processes, Geom. Funct. Anal. 7 (1997), 936-953.

[7] M. Talagrand, Regularity of Gaussian processes, Acta Math. 159 (1987), 99-149.

[8] —, The supremum of some canonical processes, Amer. J. Math. 116 (1994), 284-325.

[9] - The Generic Chaining. Upper and Lower Bounds of Stochastic Processes, Springer, Berlin, 2005.

Rafał Latała

Institute of Mathematics

Warsaw University

Banacha 2

Institute of Mathematics

02-097 Warszawa, Poland

Polish Academy of Sciences

Śniadeckich 8

E-mail: rlatala@mimuw.edu.pl

P.O. Box 21

00-956 Warszawa 10, Poland

Received October 8, 2007;

received in final form May 4, 2009 